\title{
Graft Polymerization of Acrylamide from Ultrafine Silica Particles by Use of a Redox System Consisting of Ceric Ion and Reducing Groups on the Surface
}

\author{
Norio Tsubokawa, Kazue Maruyama, Yasuo Sone, \\ and Masato SHIMOMURA* \\ Department of Applied Chemistry, Faculty of Engineering, \\ Niigata University, 8050, Ikarashi 2-nocho, \\ Niigata 950-21, Japan \\ * Advanced Technology Research Center, Technical Headquarters, \\ Mitsubishi Heavy Industries Ltd., 8-1, Sachiura 1-chome, \\ Kanazawa-ku, Yokohama 236, Japan
}

(Received November 24, 1988)

\begin{abstract}
Radical graft polymerization from ultrafine silica surface was investigated. A redox system consisting of ceric ion and the silica particles carrying reducing groups, such as alcoholic hydroxyl, amino, and mercapto groups, was capable of initiating the radical polymerization of acrylamide (AAm). The introduction of alcoholic hydroxyl groups onto the surface was achieved by the treatment of the silica with 3-glycidoxypropyltrimethoxysilane in acidic condition in water. Amino or mercapto groups were introduced onto the silica by reactions of surface silanol groups with 3-aminopropyltriethoxysilane or 3-mercaptopropyltrimethoxysilane, respectively. The initiating ability of the redox system changed, depending on the reducing groups on the silica surface, in the following order: alcoholic hydroxyl group $<$ amino group $<$ mercapto group. In the above redox polymerization, polyacrylamide was grafted onto the silica based on the propagation of the polymer from the radicals formed by the reaction of ceric ion with alcoholic hydroxyl, amino, or mercapto groups. The percentage of grafting onto the silica reached about $25 \%$. The silica obtained from the redox graft polymerization gave a stable colloidal dispersion in water.

KEY WORDS Ultrafine Particle / Silica / Radical Grafting / Redox Polymerization / Ceric Ion / Acrylamide / Silane Coupling Agent / Colloidal Dispersion/
\end{abstract}

In the preceding paper, we reported the grafting of poly(propylene glycol) onto an ultrafine ferrite particle by the reaction of hydroxyl groups on the surface with isocyanatecapped poly(propylene glycol). ${ }^{1}$ The percentage of grafting, however, was less than $15 \%$. As described in the review paper, it is required to initiate the graft polymerization from an active site (initiating group) introduced onto a particle surface to obtain polymer-grafted carbon black with a higher percentage of grafting. ${ }^{2}$ For instance, in the anionic ring-opening polymerization of $\beta$-propiolactone (PL) initiated by potassium carboxylate groups on carbon black, the percentage of grafting of poly-PL increased to about $100 \%{ }^{3}{ }^{3}$

We succeeded in the radical graft polymerization of water-soluble vinyl monomers onto carbon black initiated by a redox system consisting of ceric ions and alcoholic hydroxyl groups on the surface. ${ }^{4,5}$ In the redox polymerization of acrylamide (AAm), poly-AAm was effectively grafted from carbon black based on the propagation of the polymer from radicals formed by the reaction of ceric ions with alcoholic hydroxyl groups.

In the present paper, we report the graft polymerization of AAm from an ultrafine silica 
initiated by a redox system consisting of ceric ion and reducing groups, such as alcoholic hydroxyl, amino, and mercapto groups, introduced onto the surface. Furthermore, the initiating activity of these reducing groups for the redox graft polymerization of AAm is discussed.

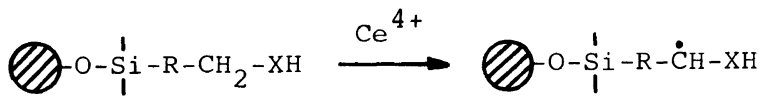

$$
\begin{aligned}
& \text { or } \\
& \bigotimes_{1}^{1} \mathrm{~S}_{i-\mathrm{R}-\mathrm{CH}_{2}-\mathrm{X}}
\end{aligned}
$$

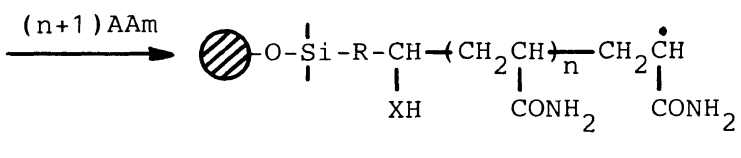

$$
\begin{aligned}
& \text { or }
\end{aligned}
$$

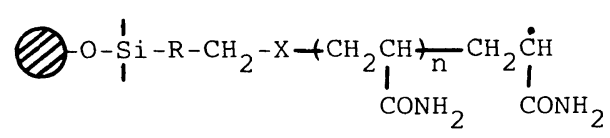

$$
\begin{aligned}
& \mathrm{XH}=\mathrm{OH}, \mathrm{NH}_{2} \text {, and } \mathrm{SH}
\end{aligned}
$$

\section{EXPERIMENTAL}

\section{Materials}

Ultrafine silica used was Aerosil 200 obtained from Nippon Aerosil Co. Its average particle size was $16 \mathrm{~nm}$ and BET specific surface area was $200 \mathrm{~m}^{2} \mathrm{~g}^{-1}$. The content of hydroxyl groups of the silica surface was determined to be 1.37 meqg $^{-1}$ by measuring volumetrically the amount of hydrogen evolved by reaction with triethylaluminium. ${ }^{6,7}$ The silica was washed with water and dried in vauo at $110^{\circ} \mathrm{C}$ before use.

3-Glycidoxypropyltrimetoxysilane (GPS), 3aminopropyltriethoxysilane (APS), and 3-mercaptopropyltrimethoxysilane (MPS) were obtained from Kanto Chemical Co., Inc. and used without further purification.

Acrylamide (AAm) was recrystallized from benzene and sublimed under reduced pressure. Guaranteed-grade ceric ammonium nitrate and nitric acid were used without further purification. Toluene was washed with concentrated sulfuric acid, refluxed over sodium, and distilled. Other reagents and solvents were also used after ordinary purification.

\section{Introduction of Alcoholic Hydroxyl Groups onto the Surface of Silica}

The introduction of alcoholic hydroxyl groups onto the silica surface was achieved by treatment with GPS in acidic conditions. ${ }^{8}$ Into a flask that contained $3.0 \mathrm{~g}$ of vacuum dried silica, $100 \mathrm{~cm}^{3}$ of a $10 \%$ aqueous solution of GPS adjusted to $\mathrm{pH} 3.5$ was added. The mixture was stirred with a magnetic stirrer at $90^{\circ} \mathrm{C}$ for $2 \mathrm{~h}$. After the reaction, the silica was filtered, washed with water, and dried in vacuo.

\section{Introduction of Amino or Mercapto Groups onto the Surface of the Silica}

The ultrafine silica carrying amino groups was prepared by reaction of the particle with APS and that carrying mercapto groups was prepared by reaction with MPS. A typical example is as follows: Into a flask that contained $3.0 \mathrm{~g}$ of silica, $100 \mathrm{~cm}^{3}$ of a $2 \%$ toluene solution of APS was added. The mixture was refluxed in a stream of nitrogen for $20 \mathrm{~h}$. After the reaction, the silica was extracted with 
methanol for $24 \mathrm{~h}$ and dried in vacuo.

\section{Polymerization Procedures}

Into a flask, $0.50 \mathrm{~g}$ of silica and $9.0 \mathrm{~cm}^{3}$ of $4.7 \mathrm{~mol} \mathrm{dm}^{-3}$ aqueous solution of AAm were charged. After deaeration of the mixture by bubbling nitrogen, $1.0 \mathrm{~cm}^{3}$ of $0.2 \mathrm{~mol} \mathrm{dm}^{-3}$ solution of ceric ammonium nitrate in $1 \mathrm{~mol}$ $\mathrm{dm}^{-3}$ nitric acid was added. The polymerization was conducted at $30^{\circ} \mathrm{C}$ with stirring by a magnetic stirrer under dry nitrogen. After the reaction, the reaction mixture was poured into a large excess of acetone to precipitate the polymer containing silica and the product was dried in vacuo at $45^{\circ} \mathrm{C}$. The conversion was determined by the following equation:

Conversion $(\%)$

$$
=\frac{\text { Precipitate }(\mathrm{g})-\text { Silica }(\mathrm{g})}{\text { AAm used }(\mathrm{g})} \times 100
$$

\section{Percentage of Grafting of Poly-AAm}

To isolate poly-AAm-grafted silica, the product was dispersed in distilled water and the dispersion was centrifuged at $3.0 \times 10^{3} \mathrm{rpm}$ until silica particles precipitated completely. The silica precipitated was dispersed again in water and centrifuged. The procedure was repeated until no polymer was detected in the supernatant solution. The percentage of grafting onto the surface was calculated by the following equation:

\section{Percentage of grafting $(\%)$}

$$
=\frac{\text { Poly-AAm grafted }(\mathrm{g})}{\text { Silica used }(\mathrm{g})} \times 100
$$

Stability of Dispersion of Poly-AAm-Grafted Silica

Poly-AAm-grafted silica $(0.30 \mathrm{~g})$ was dispersed in $100 \mathrm{~cm}^{3}$ of distilled water with a magnetic stirrer and the dispersion was allowed to stand at room temperature. After a definite time, $5.0 \mathrm{~cm}^{3}$ of dispersion liquid was taken out with a pipette and the content of silica dispersed was determined. The stability of dispersion was determined by the following equaition:

Stability of dispersion $(\%)$

$$
=\frac{\text { Silica dispersed after standing }(\mathrm{g})}{\text { Silica dispersed before standing }(\mathrm{g})} \times 100
$$

\section{RESULTS AND DISCUSSION}

Introduction of Alcoholic Hydroxyl, Amino, or Mercapto Groups onto the Silica Surface

Introduction of alcoholic hydroxyl groups onto the silica surface was achieved by hydrolysis of the glycidoxy group introduced by the coupling of GPS (eq 2). It has been reported that mildly acidic conditions $(\mathrm{pH}=3.5)$ used in coupling of GPS to silica are sufficient to convert the glycidoxy group to alcoholic hydroxyl group during the coupling reaction. ${ }^{8}$

Amino or mercapto groups can be introduced onto the silica surface by reaction of their silanol groups with APS or MPS, respectively (eq 3 and 4).

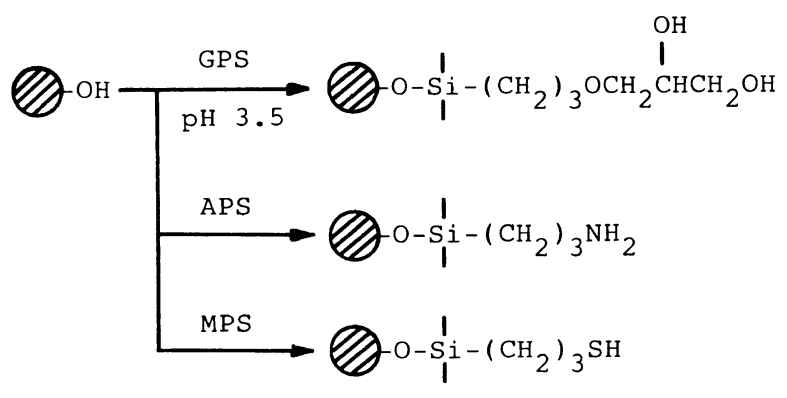


The amounts of alcoholic hydroxyl, amino, and mercapto groups introduced onto the surface were determined by elemental (carbon) analysis. The results are shown in Table I. The amount of alcoholic hydroxyl groups on the surface was estimated based on the assumption that a glycidoxy group produces two alcoholic hydroxyl groups (primary and secondary alcohol) (eq 2).

Table I clearly shows that alcoholic hydroxyl, amino, or mercapto groups can be introduced onto the silica surface. The amounts of functional groups decreased in the following order: alcoholic hydroxyl group > amino group $>$ mercapto group. Particularly, GPS was found to have high reactivity toward

Table I. Hydroxyl, amino, or mercapto groups introduced onto the surface of ultrafine particles

\begin{tabular}{lccc}
\hline & $\begin{array}{c}\text { Silane coupling } \\
\text { agent fixed }\end{array}$ & & Reducing groups \\
& meq g $^{-1}$ & & meq g $^{-1}$ \\
& 0.36 & \\
\hline Silica-R-OH & 0.31 & & 0.72 \\
Silica-R-NH & 0.26 & 0.31 \\
Silica-R-SH & 0.26 \\
\hline
\end{tabular}

a Determined by elemental (carbon) analysis.

b The amount of hydroxy groups was estimated based on the assumption that a glycidoxy group produces two hydroxy groups.

Table II. Polymerization of AAm under various conditions

\begin{tabular}{clccc}
\hline & & $\mathrm{Ce}^{4+}$ soln. $^{\mathrm{a}}$ & & Conversion $^{\mathrm{b}}$ \\
\cline { 5 - 5 } & Silica & $\mathrm{cm}^{3}$ & & $\%$ \\
\hline 1 & None & 1.0 & & 5.0 \\
2 & Untreated & - & & 0 \\
3 & Untreated & 1.0 & & 4.9 \\
4 & Silica-R-OH & - & & 0 \\
5 & Silica-R-OH & 1.0 & & 9.5 \\
\hline
\end{tabular}

a $0.2 \mathrm{~mol} \mathrm{dm}^{-3}$ of ceric ammonium nitrate solution in $1.0 \mathrm{~mol} \mathrm{dm}^{-3}$ nitric acid.

b Silica, $0.50 \mathrm{~g}$; AAm aqueous solution $\left(4.7 \mathrm{~mol} \mathrm{dm}^{-3}\right)$, $9.0 \mathrm{~cm}^{3} ; 30^{\circ} \mathrm{C} ; 24 \mathrm{~h}$. silanol groups on the silica surface under the acidic reaction conditions.

Initiation by a Redox System Consisting of Ceric Ions and Silica Carrying Alcoholic Hydroxyl Groups

It has been reported that vinyl polymerizations are initated by ceric ions in combination with organic reducing agents such as alcohols, ${ }^{9,10}$ amines, ${ }^{11}$ and thiols. ${ }^{12}$ A redox system, particularly, ceric ion/alcohol system, has been used for the grafting of vinyl monomers onto poly(vinyl alcohol) and starch. ${ }^{13}$

The polymerization of AAm was carried out by a redox system consisting of ceric ions and silica carrying alcoholic hydroxyl groups (Silica-R-OH) under various conditions. The results are shown in Table II.

The polymerization of AAm in the absence of ceric ions was not initiated by either untreated silica (No. 2) or Silica-R-OH alone (No. 4). The system consisting of ceric ions and untreated silica has no ability to initiate the polymerization (No. 3), because the conversion of the system is almost equal to that of ceric ions alone (No. 1).

On the contrary, the polymerization of AAm was found to proceed in the coexistence of ceric ions and Silica-R-OH (No. 5). These results suggest that the radical formed by the redox reaction of ceric ions with alcoholic hydroxyl groups on Silica-R-OH initiates the polymerization.

\section{Initiating Activity of a Redox System Consist- ing of Ceric Ions and Silica Carrying Reduc- ing Groups}

The effects of reducing groups on the initiating activity of redox polymerization for AAm were investigated.

Figure 1 shows the time conversion curves of the polymerization of AAm initiated by a redox system consisting of ceric ions and silica carrying reducing groups (Silica-R-OH, Silica$\mathrm{R}-\mathrm{NH}_{2}$, and Silica-R-SH). This indicates that the polymerization was initiated by Silica-R- 


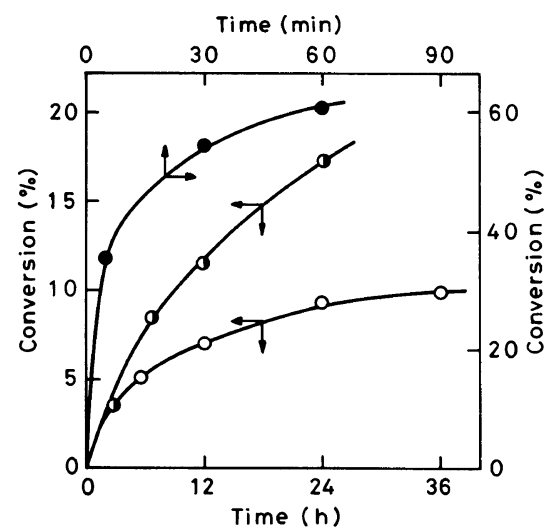

Figure 1. Polymerization of AAm initiated by a redox system consisting of ceric ion and reducing groups on silica surface. Silica, $0.50 \mathrm{~g}$; AAm aqueous solution $\left(4.7 \mathrm{~mol} \mathrm{dm}^{-3}\right), 9.0 \mathrm{~cm}^{3} ; \mathrm{Ce}^{4+}$ solution, $1.0 \mathrm{~cm}^{3} ; 30 \mathrm{C}$. (○), Silica-R-SH; (○), Silica-R- $\mathrm{NH}_{2}$; (O), Silica-R$\mathrm{OH}$.

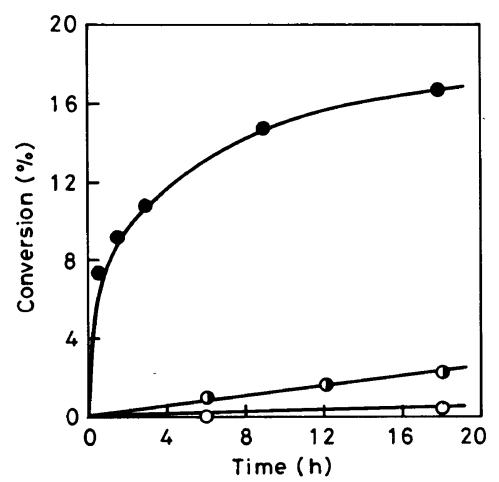

Figure 2. Polymerization of AAm initiated by a redox system consisting of ceric ion and Silica- $\mathrm{R}-\mathrm{NH}_{2}$ or Silica-R-SH at $10^{\circ} \mathrm{C}$. Polymerization conditions are given in Figure 1. (O), Silica-R-SH; (O), Silica-R- $\mathrm{NH}_{2}$; (O), Silica-R-OH.

$\mathrm{NH}_{2}$ and Silica-R-SH in combination with ceric ions as well as Silica-R-OH.

Based on the above results, it is concluded that the polymeirzation of AAm is initiated by a redox system consisting of ceric ion and reducing groups on the silica.

It is interesting that, in spite of the low content of mercapto groups, a redox system consisting of ceric ions and Silica-R-SH has very high activity for initiation. Figure 2 shows the results of the polymerization of AAm initiated by a redox system consisting of ceric ion and Silica-R-OH, Silica-R- $\mathrm{NH}_{2}$, and Silica-R-SH at $10^{\circ} \mathrm{C}$. The redox system consisting of ceric ion and Silica-R-SH was found to have high initiating activity even at $10^{\circ} \mathrm{C}$.

On the contrary, in spite of its higher content of hydroxyl groups, the system of Silica$\mathrm{R}-\mathrm{OH}$ scarcely had initiating ability of the polymerization at $10^{\circ} \mathrm{C}$. Therefore, it is concluded that the initiating activity of reducing groups on silica surface decreases in the following order: mercapto group $>$ amino group $>$ alcoholic hydroxyl group.

It is reported that in redox polymerization using $\mathrm{RCH}_{2} \mathrm{OH}$ and ceric ion, polymerization is initiated by both carbon $(\mathrm{R} \dot{\mathrm{C}} \mathrm{HOH})$ and oxygen radical $\left(\mathrm{RCH}_{2} \mathrm{O} \cdot\right) \cdot{ }^{13}$ Although little is known about the mechanism of initiation by a redox system consisting of ceric ion and amines or thiols, the difference in the initiating activity of these redox systems is due to the reducing activity of these groups.

The bond energy of $\mathrm{C}-\mathrm{H}(\mathrm{O}-\mathrm{H}), \mathrm{N}-\mathrm{H}$, and $\mathrm{S}-\mathrm{H}$ bond gave a clue for estimating the reducing activity of these groups. The bond energy of these groups is known to decrease in the following order: $\mathrm{C}-\mathrm{H}(\mathrm{O}-\mathrm{H})>\mathrm{N}-\mathrm{H}>\mathrm{S}-$ $\mathrm{H} .{ }^{14}$ However, further detailed investigation on the oxidation-reduction potentials of these reducing groups is required to estimate the reducing activity more exactly.

\section{Proof of Grafting onto Silica}

To assess the grafting of poly-AAm from the silica, the percentage of grafting was determined. The results are summarized in Table III. As shown in Table III, the percentage of grafting onto Silica-R-OH, Silica-R- $\mathrm{NH}_{2}$, and Silica-R-SH reached $10-24 \%$. To evaluate the adsorption of poly-AAm onto the silica, the silica was mixed with poly-AAm in water at $30 \mathrm{C}$ for $20 \mathrm{~h}$. The amount of the polymer remaining on the surface after the washing with water was determined to be $2.5 \%$. This value is much less than that of the polymer 
Table III. Percentage of grafting of poly-AAm from silica surface ${ }^{a}$

\begin{tabular}{|c|c|c|c|}
\hline \multirow{2}{*}{ Silica } & Time & Conversion & $\begin{array}{l}\text { Percentage } \\
\text { of grafting }\end{array}$ \\
\hline & h & $\%$ & $\%$ \\
\hline Untreated & 24 & 4.9 & 0 \\
\hline Silica-R-OH & 24 & 9.5 & 10.5 \\
\hline Silica-R-NH ${ }_{2}$ & 24 & 17.0 & 23.9 \\
\hline Silica-R-SH & 0.08 & 36.0 & 24.3 \\
\hline
\end{tabular}

a Polymerization conditions are given in Table II.

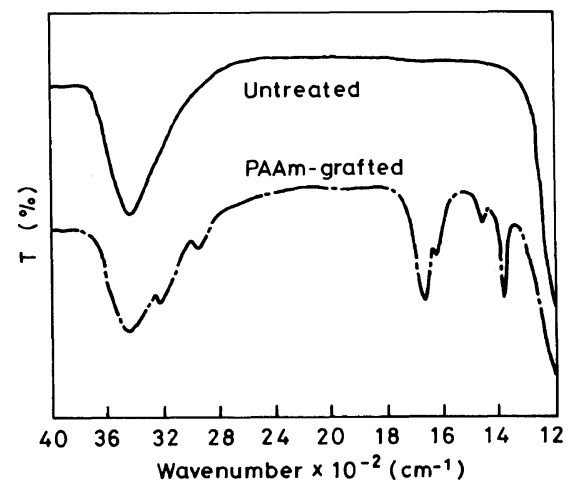

Figure 3. IR spectra of ungrafted and poly-AAmgrafted silica.

grafted onto the silica surface. During the polymerization in the coexistence of untreated silica and ceric ions, no grafting of poly-AAm was observed.

Therefore, it is concluded that poly-AAm is grafted from the silica based on the propagation of the polymer from the radical formed on the surface. The redox graft polymerization is accompanied by the formation of ungrafted poly-AAm. This may be due to a chain transfer reaction of growing polymer radical.

Figure 3 shows the IR spectra of untreated and poly-AAm-grafted silica. In the IR spectra of poly-AAm-grafted silica, a new absorption at $1668 \mathrm{~cm}^{-1}$, characteristic of amide bond of poly-AAm, and an absorption at $3190 \mathrm{~cm}^{-1}$, corresponding to stretching of the $\mathrm{N}-\mathrm{H}$ bond,

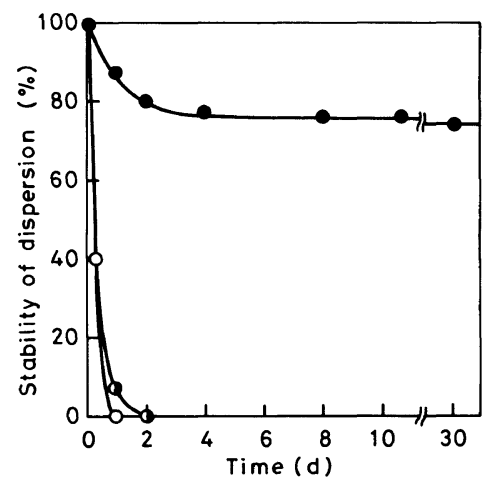

Figure 4. Stability of dispersion of poly-AAm-grafted silica in water at room temperature. (O), PAAm-grafted silica; ( ), PAAm-adsorbed silica; $(\bigcirc)$, ungrafted silica.

were observed. On the other hand, the IR spectra of silica, which was washed with water after adsorption of poly-AAm, was the same that of an untreated one. These results also indicate that poly-AAm is grafted onto the surface of silica.

\section{Stability of Dispersion of Poly-AAm-Grafted \\ Silica}

The dispersibility of poly-AAm-grafted silica in water was compared with that of untreated and poly-AAm-adsorbed ones. The results are shown in Figure 4.

Although untreated silica precipitated completely within 1 day, poly-AAm-grafted silica gave a stable colloidal dispersion in water. The stability of dispersion of silica was scarcely improved by adsorption of poly-AAm onto the surface. These results suggest that polyAAm chains grafted onto the silica surface interfere with the aggregation of silica particles. Accordingly, the dispersibility of the silica in water was remarkably improved by grafting of poly-AAm onto the surface.

Acknowledgment. This work was partly supported by the Ministry of Education, Science, and Culture of Japan under Grant (No. 62550667) to N.T., which is gratefully acknowledged. 


\section{REFERENCES}

1. N. Tsubokawa, K. Maruyama, Y. Sone, and M. Shimomura, Colloid Polym. Sci., in press.

2. N. Tsubokawa, Nippon Gomu Kyokaishi, 58, 306 (1985).

3. N. Tsubokawa, A. Funaki, Y. Hada, and Y. Sone, $J$. Polym. Sci., Polym. Chem. Ed., 20, 3297 (1982).

4. N. Tsubokawa, K. Fujiki, and Y. Sone, J. Polym. Sci., Polym. Chem. Ed., 24, 191 (1986).

5. N. Tsubokawa, K. Fujiki, and Y. Sone, J. Macromol. Sci.-Chem., A25, 1159 (1988).

6. M. Sato, T. Kobayashi, and Y. Sima, J. Catalysis, 7, 342 (1976)

7. S. Matsuda and S. Okazaki, Nippon Kagaku Kaishi,
1287 (1986).

8. F. E. Regnier and R. Noel, J. Chromatogr. Sci., 14, 316 (1976).

9. G. Mino, S. Kaizerman, and E. Rasumussen, $J$. Polym. Sci., 38, 393 (1959).

10. A. A. Katai, V. K. Kulshrestha, and R. H. Marchessault, J. Polym. Sci., C, 2, 403 (1963).

11. S. K. Saha and A. K. Chaudhuri, J. Polym. Sci., A, 1, 797 (1972).

12. G. Mino and S. Kaizerman, U.S. Patent, $2,922,774$ (1960).

13. G. Mino and S. Kaizerman, J. Polym. Sci., 31, 242 (1958).

14. J. A. Dean, Ed., "Lange's Handbook of Chemistry," McGraw-Hill Book Company, New York, N.Y., 1973. 